\title{
Simple Adaptive Synergetic Control Scheme Based on The MIT Rule of the DC Motor
}

\author{
Salomé Essiane Ndjakomo, Jacquie Thérèse Ngo Bissé, and Mathieu Jean Pierre Pesdjock
}

\section{ABSTRACT}

An adaptive synergetic control scheme of a DC motor via a DC-DC converter is presented in this article. The difficulty in implementing a synergetic control lies in the optimal choice of the control parameters. This operation becomes tedious when the number of closed loops in the control system is large. Several adaptive parameter methods are exposed in the literature, some of which are complex and others simple. A synergetic control parameter adaptation law is almost unexplored in the scientific literature. The proposed solution is an adaptation mechanism of the synergetic control parameter using the MIT (Massachusetts Institute of Technology) rule. A theoretical study carried out made it possible to highlight a law of adjustment of the current and the speed, the variation of which verifies the negative direction of the gradient of the defined cost function. Computer simulations in Matlab/Simulink show good controller performance in improving transient stability and settling time. In addition, synergetic control is an interesting tool for speed and current control of DC motor.

Keywords: Adaptive law, DC motor, MIT rule, Synergetic control.
Published Online: November 28, 2020

ISSN: $2684-4451$

DOI :10.24018/ejphysics. 2020.2.6.30

S. Ndjakomo Essiane *

Higher Technical Teachers Training College of University of Yaoundé I in Ebolowa, Department of Electrical Engineering, Cameroon.

(e-mail: salomendjakomo@gmail.com) J. T. Ngo Bissé

Higher Technical Teachers Training College of University of Yaoundé I in Ebolowa, Department of maintenance, Cameroon.

(e-mail: jacquieday13@gmail.com)

M. J. P. Pesdjock

Unité de Recherche d'Automatique et d'Informatique Appliquée, IUT Fotso Vitor de Bandjoun, Université de Dschang, Cameroon.

(e-mail: mathieuthales ${ }^{@ g m a i l . c o m) ~}$

*Corresponding Author

\section{INTRODUCTION}

The incessant evolution of technology in this century has thrown us into a world where performance is a primary objective. Generally, in industrial process control, it is still not a question of determining or adjusting adequate control strategies to maintain system inputs at desired setpoints, but rather of optimizing one or more performance criteria while respecting operating constraints. For example, the control of DC motor requires the optimisation of certain physical parameters such as speed, position, angle and current. The DC motor has several applications, namely the attraction of electric vehicles, robotic manipulators or machine tools in industry [1] and requires precision, robustness and fidelity of the control systems. In order to improve the performance of DC motors, several control schemes and methods are proposed in the literature: Proportional Integral Derivative (PID) controller is used to regulate the speed of DC motors via DC-DC converters [2]. It is the most widely used because it has a simple configuration and good performance under certain operating conditions. PID regulators can be divided into two categories [3]. PID controllers with fixed and adaptive parameters whose laws of adaptation are derived from fuzzy logic [4], neural networks, MIT rule [5], recursive least square methods [6] and other hybrid methods in the literature. However, other robust control methods such as sliding mode [7], meta-heuristic algorithm [1], fuzzy logic, neural network and many other non-linear control techniques are some of the methods for regulating the parameters of the DC machine to improve its operating performance. Despite these various methods of regulation, human satisfaction is far from being achieved. In [8], the theory of synergetic control is exploited in regulation of permanent magnet synchronous motor parameters, [9] and [10] use the same theory to control a static compensator and [11] shows that synergetic control contributes to the reduction of harmonics current injected by grid-connected photovoltaic systems. The work of [12]presents synergistic control as a strong and robust tool in the automation of robotic manipulators. It brings with notable satisfaction high quality precision and fidelity. In [13] the synergetic theory is used for indirect field-oriented control to improve the performance of an induction machine. The results presented show that the proper choice of the control parameter reduces peaks and oscillations during the transient phase. The selection of the control parameter for the synergistic control is manual. This works presents synergetic control as a robust and efficient tool for controlling industrial processes. The problem posed by synergetic control is the fateful choice of control parameters. No method on the choice of this parameter is indicated in the literature. In this study we propose an adaptive synergetic control technique based on the MIT rule and it contributes to: 
- Reduce the time needed to choose the control parameters;

- Optimize the control parameters by simple rule;

- Reduce oscillations in transient mode;

- Improve the convergence time.

This work is structured in sections as follows. Section 2 introduces the power system and its mathematical model, which takes into account the equations that describe the DC motor. In section 3 we exploit synergetic theory to derive a law for controlling the speed and current of the DC motor from the problem formulated in section 2 . The innovative and central part of our study is in section 4 . In this section we highlight the mechanism that allows the synergetic control parameters to be updated. Section 5 presents the numerical results in the Matlab/Simulink environment. Comparative analysis with the classical synergetic control is invested in order to show the contributions of the proposed control method. The last section which is section 6 is the conclusion.

\section{POWER SYSTEM MODELLING}

Let us consider Fig which describes the DC machine connected to the buck converter. The average matrix based on the study of [14] is given by the equation (1).

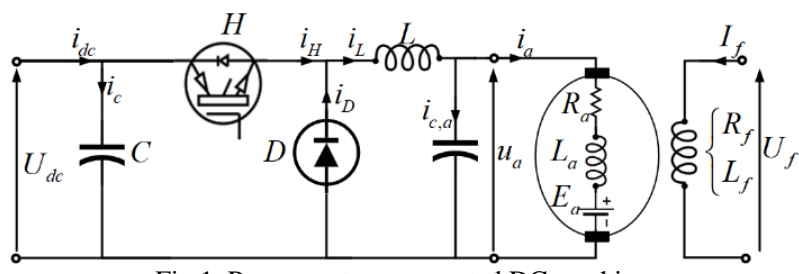

Fig.1. Power system connected DC machine.

$$
\frac{d}{d t}\left[\begin{array}{c}
i_{L} \\
i_{a} \\
u_{a} \\
\Omega \\
i_{f}
\end{array}\right]=\left[\begin{array}{ccccc}
0 & 0 & -\frac{1}{L} & 0 & 0 \\
0 & \frac{1}{L_{a}} & -\frac{R_{a}}{L_{a}} & 0 & 0 \\
\frac{1}{C_{a}} & -\frac{1}{C_{a}} & 0 & 0 & 0 \\
0 & \frac{K}{J_{t}} & 0 & -\frac{f}{J_{t}} & 0 \\
0 & 0 & 0 & 0 & -\frac{R_{f}}{L_{f}}
\end{array}\right]\left(\begin{array}{c}
i_{L} \\
i_{a} \\
u_{a} \\
\Omega \\
i_{f}
\end{array}\right]+\left[\begin{array}{c}
\frac{\alpha}{L} \\
-\frac{1}{L_{a}} \\
0 \\
-\frac{1}{J_{t}} \\
\frac{1}{L_{f}}
\end{array}\right]\left(\begin{array}{lllll}
U_{d c} & E_{a} & 1 & C_{r} & \left.U_{f}\right) \\
\end{array}\right]
$$

where $i_{L}$ is the current flowing through the inductance $\mathrm{L}, i_{a}$, $u_{a}$ are the armature current and voltage respectively, $\Omega$ the speed, $i_{f}$ and $U_{f}$ are the field current and voltage, $J_{t}$ the rotor moment of inertia, $K$ is the torque constant, $f$ is the viscous friction constant, $C_{r}$ is the load torque and $\alpha$ is the duty cycle.

Let us consider the reduced model extracted from (1) given as follow:

$$
\left\{\begin{array}{c}
\dot{x}=f(x, t)+g(x, t) u(x, t) \\
i_{a} \approx i_{L}
\end{array}\right.
$$

where $\quad x=\left[\begin{array}{ll}i_{a} & \Omega\end{array}\right]^{T}$ are the state vector, $f(x, t) \triangleq\left[\begin{array}{c}-\frac{u_{a}}{L} \\ -\frac{f \Omega+C_{r}}{J_{t}}\end{array}\right], \mathrm{g}(x, t) \triangleq\left[\begin{array}{c}-\frac{U_{d c}}{L} \\ \frac{K}{J_{t}}\end{array}\right]$ and $u=\left[\begin{array}{ll}\alpha & i_{a}\end{array}\right]$ is the control vector. Equation (2) is the formulation of the problem.

\section{SYNERGETIC CONTROL DESIGN}

In order to achieve the objective of synergetic control, the macro-variable of the closed-loop system is defined as follows:

$$
\Psi(x, t)=\phi(x, t)=x^{*}-x
$$

$\Psi$ is the macro-variable, $x^{*}$ is the reference vector and $\Phi$ is the objective function that must satisfy the constraint defined by equation (4) [10], [15]

$$
\phi(x, t)=0
$$

In order to satisfy the requirement imposed by (4), the constraint on the evolution of macro-variable is defined as follows [9], [16]:

$$
\lambda \dot{\Psi}(x, t)+\Psi(x, t)=0
$$

where the control parameter $\lambda$ indicates the speed of convergence of the closed loop system to the domain defined in (4). Substituting (2) with the time derivative of (3) gives equation (6).

$$
\dot{\Psi}(x, t)=\dot{x}^{*}-f(x, t)-g(x, t) u(x, t)
$$

By substituting (6) in (5), the following control vector is deduced:

$$
u(x, t)=g^{-1}(x, t)\left\{\dot{x}^{*}-f(x, t)+\lambda^{-1} \Psi(x, t)\right\}
$$

The control law given in (7) is used to regulate the speed and current of the DC motor. However, the function $\lambda(t)$ is often defined as constant and chosen manually. Therefore, an adaptive law of this parameter by the MIT rule will be proposed here.

\section{ADAPTIVE LAW DESIGN}

The adaption mechanism based on the MIT rule here is used to adjust the $\lambda(t)$ parameter of the control law defined in (7). The cost function of this rule which minimises the micro-variable defined in (3) is:

$$
j(\lambda(t))=\frac{1}{2} \psi^{2}(x, t)
$$

For this, it is preferable to adjust the parameter $\lambda$ in the negative direction of the gradient of $j$ as shown in equation (9).

$$
\frac{\partial \lambda(t)}{\partial t}=-\gamma \frac{\partial j(x, t)}{\partial \lambda(t)}=-\gamma \psi(t) \frac{\partial \psi(x, t)}{\partial \lambda(t)}
$$


where $\lambda(t)=\left[\begin{array}{ll}\lambda_{1}(t) & \lambda_{2}(t)\end{array}\right]^{T}>0$ and $\gamma>0$. By transforming (9) into (10), we deduce the solutions given by (10).

$$
\begin{aligned}
& \frac{\partial \lambda(x, t)}{\partial t}=-\gamma \psi(x, t) \frac{\partial \psi(x, t)}{\partial u(x, t)} \cdot \frac{\partial u(x, t)}{\partial \lambda(t)} \\
& \left\{\begin{array}{l}
\frac{\partial \psi(t)}{\partial u(x, t)}=g(x, t) \lambda(t) \\
\frac{\partial u(x, t)}{\partial \lambda(t)}=-\psi(x, t) g^{-1}(x, t) \lambda^{-2}(t)
\end{array}\right.
\end{aligned}
$$

For the convergence condition to be verified, $\lambda$ must be chosen as indicated by (12).

$$
\frac{\partial}{\partial t}(\lambda(t))^{2}=2 \gamma(\psi(x, t))^{2}
$$

By substituting equation (12) in equation (9) we verify the direction of the negative gradient given as follows.

$$
\left\{\begin{array}{l}
\frac{\partial j(x, t)}{\partial \lambda(t)}=-\frac{\psi^{2}(x, t)}{\lambda(t)} \\
\frac{\partial \psi(x, t)}{\partial \lambda(t)}=-\frac{\psi(x, t)}{\lambda(t)}
\end{array}\right.
$$

\section{RESUlTS AND DISCUSSIONS}

In order to validate the adaptive synergetic control law, the numerical simulation results under the Matlab/Simulink environment are presented in Fig. 2 and 3. The performance of the system has been obtained under speed variation as described in fig. 3(a). The simulation parameters are given in Table 1 and Fig. 2 shows the parameters $\tau_{1}$ and $\tau_{2}$ adapted by the MIT rule in Fig. 2(a) and 2(b) respectively. Fig. 3 shows a detailed comparison between the two-control law. The variation and evolution of the DC motor speeds shown in Fig. 2(a) position the adaptive synergetic control as the best in convergence speed, accuracy and settling time. Zooms in Fig. 3(a) present an adaptive synergetic control as a tool for improving transient stability in DC motor speed control. The evolution of the torque presented in Fig. 3(c) is proportional to the current in Fig. 3(b). A considerable improvement in transitional regime trough of the proposed order is observed. It cancels the oscillations and improves the system response. Fig. 3(d) shows the armature voltage response of the motor so the benefits are identical to those shown in Fig. 3(b) and 3(c). The adaptive control proposed in this article provides a satisfactory reduction in the oscillations of the conventional control and contributes to improved system performance. Indeed, the adaptive method by the MIT rule of the $\lambda$ parameter improves the performance of the synergetic controller while reducing oscillations, improves settling time, reduces noise, increases robustness, and makes the system more accurate and faithful. The particularity of the proposed control law lies in the search of the optimal values of $\tau_{1}$ and $\tau_{2}$ for the best system performance.
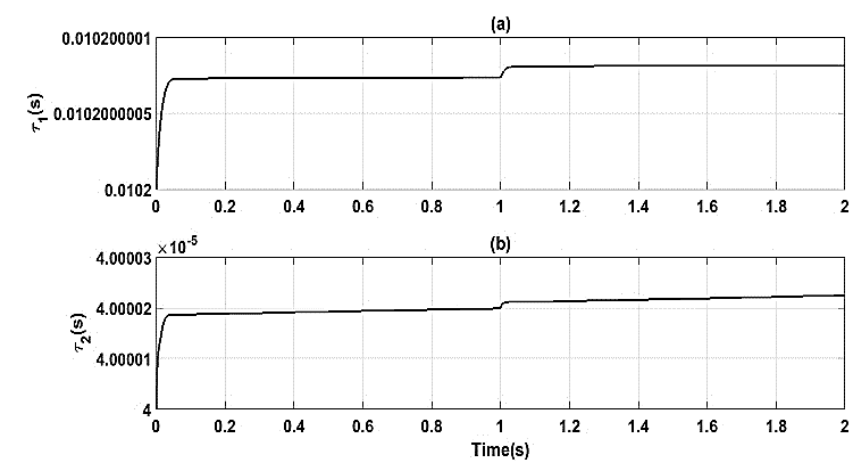

Fig. 2. Convergence rate adapted under variation speed.

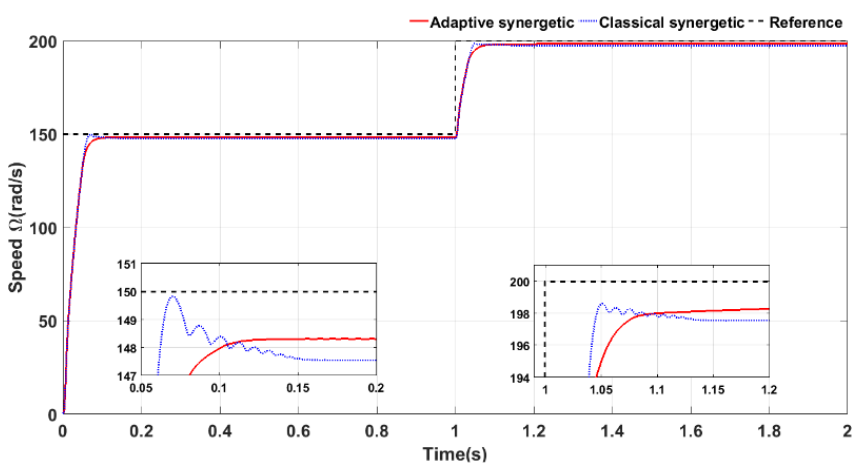

(a)

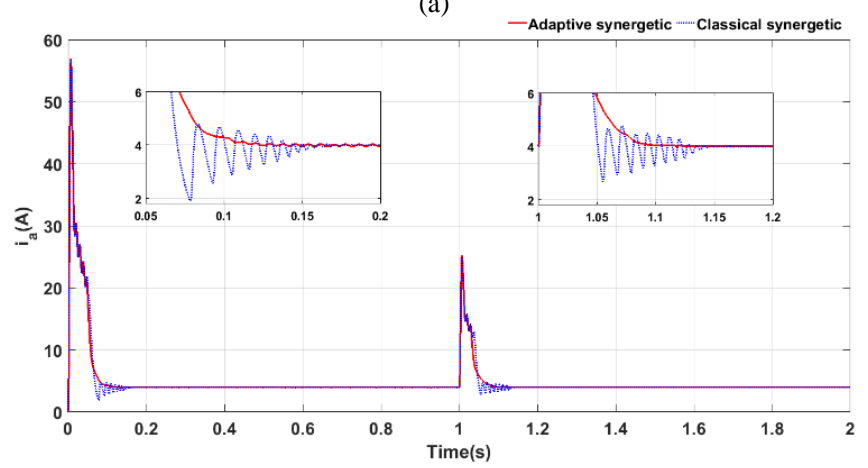

(b)

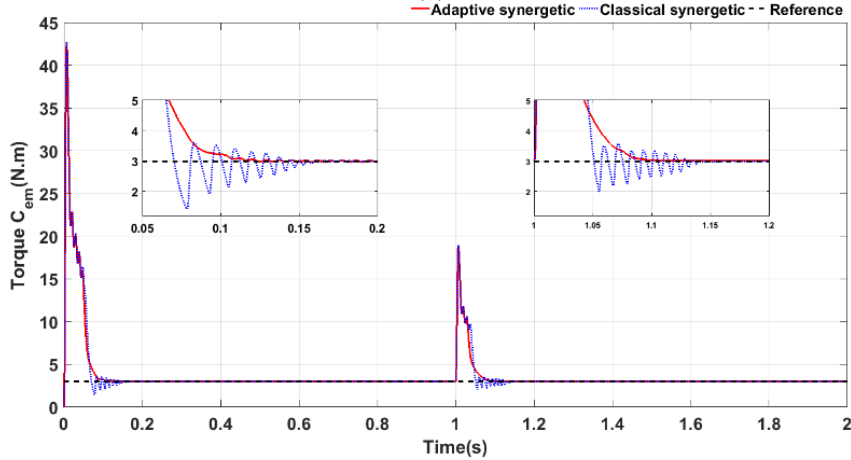

(c)

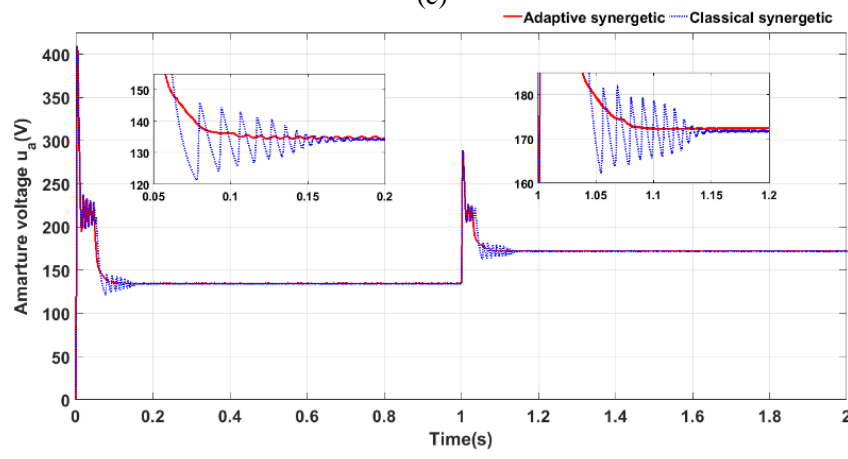

(d)

Fig. 1. System performances under varying speed: (a) Speed $\Omega$; (b); Armature current $\mathrm{i}_{\mathrm{a}}$; (c) Torque $\mathrm{C}_{\mathrm{em}}$; (d) Armature voltage $\mathrm{u}_{\mathrm{a}}$. 


\begin{tabular}{cccccc}
\multicolumn{6}{c}{ TABLE 1: SIMULATION PARAMETERS } \\
\hline $\boldsymbol{R}_{\boldsymbol{a}}$ & $\boldsymbol{L}_{\boldsymbol{a}}$ & $\boldsymbol{f}$ & $\boldsymbol{K}$ & $\boldsymbol{I}_{\boldsymbol{a}, \boldsymbol{n}}$ & $\boldsymbol{J}$ \\
\hline $\mathbf{5 . 5} \boldsymbol{\Omega}$ & $0.0119 H$ & $4.051 \times 10^{-4} \mathrm{Nms}$ & 0.859 & 4.50 & 0.00705 \\
$\boldsymbol{\gamma}$ & $U_{\boldsymbol{a}}$ & $P$ & $\tau_{1}$ & $\tau_{2}$ & $\mathrm{kgm}^{2}$ \\
$\mathbf{1 0}^{-\mathbf{8}}$ & $220 \mathrm{~V}$ & $1 \mathrm{~kW}$ & $5.38 \times 10^{-3}$ & $27.8 \times 10^{-7}$ & \\
\hline
\end{tabular}

\section{CONCLUSION}

In this paper, an adaptive synergetic control scheme of a DC motor via a DC-DC converter is presented. The implementation of an adaptation law using the MIT rule has made it possible to optimize the control parameters and remove the difficulty of choosing these parameters. The theoretical study carried out made it also possible to highlight a law of adjustment of the current and the speed, the variation of which verifies the negative direction of the gradient of the defined cost function. Computer simulations were made to verify the performance of the solution and shows how the system can be improved under the speed variation. Numerical simulations in Matlab/Simulink shows good controller performance in improving transient stability, settling time and reduce oscillation. In addition, synergetic control is an interesting tool for speed and current control of DC motor. The improvement of these performances allows to reduce losses, winding heating, acoustic noise and optimization of the motor efficiency. The proposed method can find application in the control of systems connected to the electrical network, in the control of asynchronous machines. Our future work focuses on the experimental implementation of synergetic control in order to verify this theory in DC motor control.

\section{ACKNOWLEDGMENT}

Our thanks go to Miss Fouda Bella Regine for reading and editing this article. The authors gratefully acknowledge the anonymous reviewers and the Editor for several relevant and stimulating remarks contributing to improving the quality of the paper.

\section{REFERENCES}

[1] R.-M. Alejandro, V.-C. Miguel Gabriel and A.-P. Mario, "An adaptive control study for the DC motor using meta-heuristic," Soft computing, Springer, vol. 23, pp. 889-896, 2017.

[2] R. Nagarajan, S. Sathishkumar, K. Balasubramani, K. Boobalan, S. Naveen and N. Sridhar, "Chopper fed speed control of DC motor using PI controller," IOSR Journal of Electrical and Electronics Engineering (IOSR-JEEE), vol. 11, no. 3, pp. 65-69, 2016.

[3] P. Swarnkar, K. Jain Shailendra et N. R.K, «Comparative analysis of MIT rule and Lyapunov rule in model reference adaptive control scheme,» Innovative Systems Design and Engineering, vol. 2, $\mathrm{n}^{\circ} \% 14$, pp. 154-163, 2011.

[4] H. Zulfatman, M. Ahzen Habibidin, N. Alif et Mardiyah, «Adaptivefuzzy-PID controller based disturbance observer for DC motor speed control,» Proc. EECSI, pp. 19-21, 2017.

[5] T. Aleksei, A. Baris Baykant, G. Emmanuel, P. Eduard and Y. Celaleddin, "Model reference adaptive control scheme for retuning method-based fractional-order PID control with disturbance rejection applied to closed-loop control of a magnetic levitation system,"' Journal of Circuits, Systems, and Computers, vol. 27, no. 11, p. 29, 2018.

[6] S. Suwandi Dwi, F. Fahmi, W. Nanda Avianto et Y. Feri, «Design and implementation of adaptive PID controller for speed control of DC motor.,»15th Intl. Conf. QiR: Intl. Symp. Elec. and Com. Eng,IEEE, pp. 169-183, 2017.

[7] M. Uma, B. Y.S Kishore et A. K, «Sliding mode speed control of a DC motor,» International Conference on Communication Systems and Network Technologies, IEEE, pp. 387-391, 2011.

[8] W. Tao, L. Jikun et L. Yuwen, «Synergetic control of permanent magnet synchronous motor based on load torque observer,» Journal of Systems and Control Engineering, pp. 1-14, 2018.

[9] F. Rehan et M. Choudhry, «Design of non-linear static Var compensator based on synergetic control theory,» Electric Power Systems Research, vol. 151, p. 243-250, 2017.

[10] H. Ran, A. Qian, Z. Yuchao, J. Ziqing et Y. Zhiwen, «Design of a distributed non-linear stabiliser in MG using synergetic control theory,» IET Generation, Transmission \& Distribution, pp. 1-11, 2018.

[11] M. J. P. Pesdjock, J. R. Mboupda Pone, G. Kenne et L. L. Sonfack, «Contribution of synergetic control to the minimization of harmonics currents injected for grid connected photovoltaic systems,» SN Apllied Sciences, vol. 2, $\mathrm{n}^{\circ} 1: 1396,2020$.

[12] L. Chi-Hua et H. Ming-Ying, «A finite time synergetic control scheme for robot manipulators,» Computers and Mathematics with Applications, p. 1163-1169, 2012.

[13] Z. Yu, J. Zhenhua et Y. Xunwei, «Indirect Field-Oriented Control of Induction,» IEEE Power and Energy Society General Meeting Conversion and Delivery of Electrical Energy in the 21st Century, pp. 1-7, 12 August 2008.

[14] J. López, S. Seleme Jr., P. Donoso, L. Morais, P. Cortizo and M. Severo, "Digital control strategy for a buck converter operating as a battery," Solar Energy, no. 140, p. 171-187, 2016.

[15] V. Gennady E., P. Ndrey N., R. Ivan A et M. Alexey S, «Adaptive power saving control for traction asynchronous electrical drive: synergetic approach,» 2014 IEEE International Energy Conference (ENERGYCON), pp. 1446-1453, 2014.

[16] N. Junkang, L. Chongxin, L. Kai et P. Xia, «Variable speed synergetic control for chaotic oscillation,» Nonlinear Dynamic, p. 681-690, 2014.

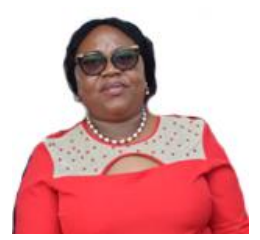

Ndjakomo E. Salomé was born on May 14, 1974 in Yaoundé, Cameroon. She is a Ph.D holder from the IBN Tofail Morocco University. Her research interests and relate to Electrical power engineering and power systems modelling. Senior Lecturer and Director of the ENSET of Ebolowa, Cameroon and Head of Department in Electrical Engineering.

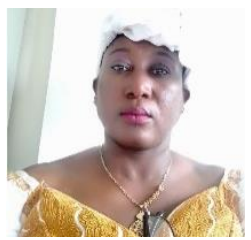

Ngo Bissé J. T. was born on October 3, 1969 in Bibia, Cameroon. She holds a Ph.D. in Telecommunications with a major in Information Theory from the Moscow Institute of Energy. Lecturer and Head of Maintenance Department at ENSET Ebolowa since 2018.

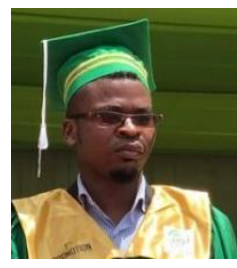

Pesdjock Mathieu J.P. was born on October 15, 1992 in Yaoundé, Cameroon. Master degree holder in Electrical Engineering from the University of Dschang in 2016. Holder of a teacher's diploma of technical and vocational high schools obtained at the ENSET of Ebolowa in 2019 and major of the whole promotion. His research interests include modelling and control power systems and system identification. 